\title{
HISTORIA DE LA PROBABILIDAD EN ESPAÑA
}

\author{
F. JAVIER MARTIN PLIEGO \\ Universidad Autónoma de Madrid
}

\section{INTRODUCCIÓN}

Es generalizada la idea de que la revolución probabilística no se produjo en España hasta después de 1930 y que nuestro pais fue un verdadero páramo donde se ignoraban las aplicaciones que la nueva metodología estadística, basada en los modelos de probabilidad que se habían desarrollado a lo largo de los dos últimos siglos en Europa, ofrecía a las ciencias sociales, en general, y a la demografía, a las compañías de seguros, a la especulación en Bolsa, a las actividades industriales y comerciales, etc. Este trabajo tiene la pretensión de poner de manifiesto el interés que algunos científicos españoles se tomaron para propagar estos conocimientos por su gran utilidad desde los inicios del surgimiento del Cálculo de Probabilidades, aunque no supieran (o no pudieran) crear una escuela que diera continuidad a sus aislados esfuerzos, intentos que también chocaron con la secular desidia de nuestra ciencia matemática a la que incomodaba cualquier conato de modernización.

En la génesis de la Estadística como ciencia se observan dos corrientes cronológicamente diferenciadas que arrancan de épocas muy antiguas y que, aun teniendo algunos contactos esporádicos en los siglos XVIII y XIX, no se fusionan, definitivamente, hasta este siglo. Dichas corrientes son, por una parte, la Estadistica como ciencia de la elaboración de estadísticas, cuyos orígenes los podemos situar en la conformación de las primeras sociedades humanas con la necesidad de efectuar recuentos de la población, catastros 
de sus tierras y censos de sus ganados y, por otra parte, el Cálculo de Probabilidades que aparece ligado al estudio de los juegos de azar.

Antes de iniciar el estudio de la evolución del Cálculo de Probabilidades en nuestro país, recordemos que los primeros escritos sobre juegos de azar empiezan a ver la luz en el Renacimiento: En Luca Pacioli (1445-1509), Cardano (1501-1576) y Galileo (1564-1642) encontramos pequeños opúsculos que versan sobre la resolución de determinados problemas planteados en los juegos de azar (principalmente en los juegos de dados).

Estos son antecedentes de lo que se considera realmente como el nacimiento del Cálculo de Probabilidades: la correspondencia que mantuvieron Blaise Pascal (1623-1662) y Pierre de Fermat (1601-1665) a raiz de ciertos problemas planteados al primero por Antoine Gombalt (1610-1685), Caballero de Méré. Esta correspondencia ${ }^{1}$ no presentaba una exposición sistemática de lo que Pascal denominó "Geometría del azar», por lo que el primer manual editado, relativo al Cálculo de Probabilidades, es el de Christian Huygens (1620-1699) titulado De Ratiociniis in ludo aleae ${ }^{2}$, que aparece en 1656.

\section{SIGLO XVII: EL SIGLO DE LAS IDEAS}

Es en esta época donde surge el primer español que se preocupa por la aplicación de los cálculos aritméticos a la resolución de problemas derivados de los juegos de dados. Juan Caramuel (1606-1682), teólogo cisterciense, nacido en Madrid, estudió en las Universidades de Alcalá y de Salamanca y se doctoró en la de Lovaina. Hombre típico del Renacimiento, con vasta cultura, se preocupó por los más variados temas: por las matemáticas en general, con aportaciones a la teoría de los cologaritmos, por la astronomía, por la ciencia de la meteorología, por la arquitectura...

En una de sus obras titulada Mathesis biceps ${ }^{3}$, publicada en 1670 , se recoge un breve tratado de 24 páginas bajo el nombre de Kybeia, quae combinatoriae genus est, de alea et ludis fortunae serio disputans. En esta Kybeia, término griego que hace referencia a los juegos de dados, efectúa un detenido análisis de cuestiones de juegos y apuestas aplicando la teoría combinatoria. «La Kybeia consta de cuatro artículos y una Diatribe, estando cada artículo

\footnotetext{
1 Una versión en español puede encontrarse en De Mora Charles, M. (1989): Los Inicios de la teoria de la probabilidad. Siglos XVI y XVII, Bilbao, Universidad del Pais Vasco.

2 De Mora Charles, M.: Los inicios de la teoría de la probabilidad.

3 Caramuel, J. (1670): Mathesis biceps (vetus et nova), Campania.
} 
dividido en varios números» ${ }^{4}$. En ellos, Caramuel, en primer lugar, y, posiblemente, para tranquilizar su conciencia cristiana, incompatible en la época con el recreo lúdico, establece que todos los juegos deben ser justos. En este sentido dice: «Para que se pueda guardar esta equidad (en el juego) es necesario que el dinero sea correspondiente al peligro, de forma que los que se exponen a un peligro semejante puedan conseguir igual dinero, pero, por el contrario, los que se exponen a un peligro desigual puedan conseguir un dinero desigual, de forma que debe depositar una cantidad menor el que se expone a un peligro mayor $y$, por el contrario, debe exponer una cantidad mayor el que se expone a un peligro menor» 5 .

Caramuel describe diferentes juegos de tabas y dados y otros juegos, y se centra en la resolución del problema del reparto del fondo de apuestas cuando el juego se interrumpe antes de su conclusión, siguiendo la tradición que inician los precursores de la probabilidad ${ }^{6}$, y que muchos autores posteriores, no solamente Caramuel, recogen. También establece el teorema de las probabilidades compuestas o regla de la multiplicación. En las últimas diez páginas de su texto, a las que denomina Diatribe, reproduce la obra de Huygens, aunque la atribuye, erróneamente, al astrónomo danés Longomontano.

Las aportaciones de Caramuel a la teoría de la probabilidad las reconocen Todhunter ${ }^{7}$, Keynes ${ }^{8}$ y Hald ${ }^{9}$, aunque no destacan que, realmente, la obra de Caramuel es el segundo tratado sobre la probabilidad que se publica en el mundo después del de Huygens.

Parece ser que la teoría del probabilismo fue iniciada en el siglo Xvi por el teólogo dominico Bartolomé Medina (1528-1580). Esta doctrina moral intentaba responder a la cuestión si se debe o no obrar en contra de una ley en caso de duda razonable, estableciendo que es lícito hacerlo siempre que la decisión se base en una opinión sólidamente probable (en el sentido de aprobable o razonablemente aceptable), aun cuando las opiniones contrarias sean tan probables o más que aquélla. Esta corriente ideológica fue seguida por la mayoría de los moralistas jesuitas de la época, puesto que su acepta-

" Garma Pons, S. (1982): «La combinatoria y las probabilidades en el siglo XVII, según Caramuel», Anuario Juridico Escurialense, núm. XIV, Real Colegio Universitario Maria Cristina, Madrid, pp. 331-364.

SCaramuel, J.: Mathesis biceps, p. 973.

6 Véase una breve historia de la teoría de la probabs

L. (1995): Estadistica I: Probabilidad, Madrid, AC, pp. $13-25$.
7 Todhunter, I. (1865): $A$ history of the mathematical theory of probability from the time of Pascal to that of Laplace, Londres, Macmillan.

8 Keynes, J. M. (1921): Treatise on probability, Londres, Macmillan. Wiley. 
ción facilitaba la aplicación de la casuística que muchos de dichos moralistas propugnaban.

Contra esta forma de actuar se rebelaron los «solitarios» de Port-Royal que, bajo la inspiración jansenista, defendían un mayor rigor en las actitudes morales y religiosas ${ }^{10}$. Para exponer sus criterios utilizaron el vehiculo de las Cartas Provinciales que encargaron escribir a Blaise Pascal ${ }^{11}$.

Pero no solamente fueron los jansenistas los que reclamaron la vuelta a posiciones menos relajadas, sino que también los dominicos y algunos jesuitas, minoritarios, reclamaron el abandono de la teoría del probabilismo y su sustitución por la del probabiliorismo, por la cual sólo era lícito actuar en contra de ley si se disponia de una opinión sólidamente fundada que fuera más probable que las contrarias.

Es en el seno de esta polémica donde aparece una nueva figura destacada en esta historia de la probabilidad, la del teólogo jesuita Tirso González de Santalla (1624-1705), catedrático de Teología de la Universidad de Salamanca, que abrazó la causa del probabiliorismo enfrentándose a la mayoría de sus compañeros y, lo que era más grave para su época, a sus superiores. En su cruzada el P. González no estuvo nunca solo ni fue el primero en defender un mayor rigor en los dictámenes de los confesionarios y en las orientaciones de los fieles ${ }^{12}$, pero sí fue el más tenaz en la lucha contra el probabilismo que había contagiado a la mayoría de los sacerdotes de su Compañia. También es a él a quien se le debe el estudio más profundo sobre este tema.

La publicación de su Fundamentum Theologiae Moralis, id est, Tractatus Theologicus de Recto Usu Opinionum Probabilium ${ }^{13}$ tuvo una gestación tan peculiar que demuestra la tenacidad y la solidez de sus propias ideas. La primera versión que quiso publicar data de 1672, pero fue desaprobada por los censores de la Compañia de Jesús en Roma. La segunda la escribió en 1680 y tampoco tuvo la preceptiva autorización. Parece que necesitó llegar a ser prepósito ge-

\footnotetext{
10 Véase Hacking, I. (1995): El surgimiento de la probabilidad, Barcelona, Gedisa.

11 Pascal, B. (1983): Obras. Pensamientos. Provinciales. Escritos científicos. Opúsculos y Cartas, Madrid, Alfaguara.

12 Véase Astraín, A. (1920): Historia de la Compañia de Jesús en la asistencia de España, vol. VI, Administración de Razón y Fe, Madrid.

13 González.de Santalla, T. (1694): Fundamentum Theologiae Moralis, id est, Tractatus Theologicus de Recto Usu Opinionum Probabilium, Roma. Jo. Jacobi Komarek. Existen otras ediciones bajo el mismo título en Nápoles. Dom. Ant. Parrino \& Michaelis Aloysii Mutti, 1694; en Zaragoza, Dio. nisium Dormer, 1694. También se editaron extensas sinopsis en Amberes, Amsterdam, Venecia, etc., bajo el titulo De Recto Usu Opinionum Probabilium. Una sinopsis más reciente puede encontrarse en Theologiae Cursus Completus, t. XI, París, Apud Editores, 1839.
} 
neral de la Compañía de Jesús para que, con permiso papal y aun en contra del deseo de sus súbditos, por fin, la tercera versión viera la luz en 1694 . Tal era su obstinación para que su trabajo se difundiera que hizo imprimir en $\mathrm{Di}$ llingen en 1691 un breve tratado intitulado Tractatus succinctus de recto usu opinionum probabilium, aunque la controvesia suscitada en el seno de la Compañía con los probabilistas impidió su difusión.

Desde el punto de vista de nuestro propósito en este trabajo debemos destacar las ideas seminales que luego influyeron en el desarrollo de la Probabilidad en su faceta epistemológica: En primer lugar, la insistencia en la gradualidad de la probabilidad; en segundo lugar, el análisis del equiprobabilismo, germen del principio de la razón insuficiente o principio de indiferencia; por último, y lo que es más reseñable, su anticipación al concepto de probabilidad subjetiva que se forma en la mente de las personas y que debe ser preferible a la opinión probable emitida por autoridad competente. En este sentido manifiesta: «Ninguna autoridad de doctores, ningún fundamento me puede mover a mí, a juzgar por verdadera una opinión, si a mí me parece menos verosimil que la sentencia contraria, siendo enteramente repugnante de suyo, que el entendimiento abrace como verdadero lo que se le representa menos verosímil que lo contrario».

\section{UNA TRADUCCIÓN CASUAL}

Tenemos que avanzar más de un siglo para encontrar un texto sobre probabilidad editado en castellano. En este caso se trata de una traducción: José Clavijo y Faxardo (1707-1806), al cual inmortalizó Goethe por su disputa de trasfondo amoroso con el caballero francés Caron de Beaumarchais en su drama «Clavijo», tradujo, como vice-director del Real Gabinete de Historia Natural, la enciclopédica Historia Natural de George Louis de Leclerc, conde de Buffon (1707-1788), recogiéndose en el Tomo VI de la edición española de $1788^{14}$ el Ensayo de Aritmética Moral que apareció en 1777 en el cuarto volumen del Suplemento a la Historia Natural, junto con sus trabajos relativos a la elaboración de tablas de mortalidad.

En el segundo párrafo de su Ensayo Buffon manifiesta ${ }^{15}$;

«Mi objeto en este ensayo es medir las cosas inciertas y dar algunas reglas para apreciar las relaciones de verosimilitud, los grados de probabilidad, el va-

14 Buffon (1788): Historia Natural. General y Particular, t. VI, Madrid, Viuda de Ibarra, Hijos y compañia.

15 Buffon: Historia Natural, t. VI, pp. 239-240. 
lor de los testimonios, la influencia de las casualidades y el inconveniente de los riesgos, y también para formar juicio del valor real de nuestros temores $y$ de nuestras esperanzas.» Además de una serie de consideraciones filosóficas sobre el alcance del concepto de probabilidad, Buffon considera que los sucesos que tengan una probabilidad extremadamente pequeña de ocurrencia pueden considerarse como sucesos «moralmente» imposibles, y estudia una solución alternativa a la que da Daniel Bernoulli en la conocida Paradoja de San Petersburgo.

Por último, efectúa una aplicación de la geometría al Cálculo de Probabilidades en la resolución de su famoso "problema de la aguja»: Se deseaba conocer la probabilidad de que una aguja de coser tirada hacia el aire cortara al caer alguna de las aristas de las baldosas del suelo o, por el contrario, quedara integramente dentro de una baldosa. Este juego se denominaba en Francia «franc carreau» y también se ejercitaba en esa época en Andalucía bajo el nombre de ladrillejo.

El resultado que se obtiene en la determinación de la probabilidad requerida depende de las longitudes de la baldosa, de la longitud de la aguja y del número $\pi$, lo que permite experimentalmente obtener el valor de $\pi$ a través de la estimación de esta probabilidad por la frecuencia relativa del número de casos en que la aguja corta alguna arista. Esta consecuencia ha hecho que el «problema de la aguja de Buffon» sea un ejemplo clásico que incluyen la mayoría de los textos actuales ${ }^{16}$.

\section{LA PROBABILIDAD EN LOS TEXTOS DE MATEMÁTICAS}

A finales del siglo xviI, y a diferencia de lo ocurría en la vecina Francia o en Inglaterra, el Cálculo de Probabilidades seguía sin ser estudiado y cultivado en nuestro país. No existía investigación y, por tanto, tampoco se crearon círculos de científicos donde de discutieran y comunicaran los nuevos adelantos. Sólo podemos constatar el empeño de algunos pocos matemáticos en difundir los conceptos y aplicaciones de la probabilidad. En esta época y a comienzos del siglo XIX aparecieron pequeños opúsculos dentro de manuales dedicados a la matemática aplicada. Este es el caso de los tres textos que comentamos a continuación.

16 Por ejemplo, puede verse Martin Pliego, J., y Ruiz-Maya, L.: Estadistica I: Probabilidad, pp. 344-345. 
Tadeo Lope y Aguilar (1753-1800), militar y catedrático de Matemáticas del Real Seminario de Nobles de Madrid, publica en 1795 el Tomo segundo de su Curso de Matemáticas ${ }^{17}$, donde incluye un verdadero tratado de Cálculo de Probabilidades que desarrolla en tres capitulos a lo largo de 118 páginas. Aunque inspirado en la obra de Abraham De Moivre (1667-1754) The Doctrine of Cbances $^{18}$, presenta ciertas singularidades que quisiera poner de manifiesto:

a) Además de definir acertadamente los conceptos de probabilidad, esperanza de un suceso, sucesos independientes o dependientes, establece, a modo de axiomática ${ }^{19}$, las ideas de equiprobalidad, esperanza de una variable y la necesidad que el juego sea justo.

b) Fija la regla de la multiplicación para determinar la probabilidad de sucesos compuestos, estableciendo que, si éstos son independientes, también dicha regla se aplica al cálculo de la probabilidad de sus complementarios.

c) Realiza un completo análisis para el cálculo de probabilidades con sucesos dicotómicos que se repiten en n pruebas.

En la página 339 de su tratado advierte de la necesidad de establecer previamente el espacio muestral, de la ventaja que supone, en determinados casos, el calcular la probabilidad del suceso complementario al deseado, y de cómo un suceso compuesto debe descomponerse en sus casos elementales para, mediante la regla de la suma, determinar su probabilidad. Con el examen de 24 ejercicios relativos a juegos de azar culmina el capitulo I de este tratado. En los dos capítulos siguientes, siguiendo la citada edición del texto de De Moivre, expone las bases actuariales para el estudio de rentas vitalicias y seguros de vida.

Benito Bails (1730-1797) incluye, a partir de la tercera edición de su curso de matemáticas ${ }^{20}$, un breve apéndice sobre los principios de las probabilidades, pues dice ${ }^{21}$ : «me he quedado lleno de admiración al ver como han manejado este asunto Matemáticos de naciones extrangeras....He procurado ponerla

17 Lope y Aguilar, T. (1795): Curso de Matemáticas para la ensenal, pp. 327-444 del Real Seminario de Nobles de Madrid, t. II, Madrid, Impren. Londres, A. Millar.

18 De Moivre, A. (1756): The Doctrine of Chances. 3.

19 Lope y Aguilar, T.: Curso de Matematíática de la Real Academia de San Fernando, t. I, 4." ed.,

20 Bails, B. (1805): Principios Madrid, Hija de Joaquin Ibarra.

21 Bails, B.: Principios de Matemática, p. ij del prólogo. 
(la probabilidad) de modo que ni espante ni preocupe». Realmente sólo dedica siete páginas escasas a la probabilidad, ocupando la mayoría del texto en la resolución de cuatro ejercicios muy elementales.

José Mariano Vallejo (1779-1846) llegó a ser catedrático de Matemáticas del Real Seminario de Nobles de Madrid y de la Real Academia de Bellas Artes de San Fernando; entre sus importantes publicaciones editó en 1819 un Compendio de Matemáticas 22 en cuyo Tomo segundo incluyó un capítulo sobre el «arte conjetural o teoría de las probabilidades», adoptando la denominación que Jacques Bernoulli (1654-1705) dio a su manual de Cálculo de Probabilidades Ars Conjectandi como contraposición al Ars Cogitandi oLógica de los jansenistas de Port-Royal Antoine Arnauld y Pierre Nicole ${ }^{23}$. Vallejo, a lo largo de las 12 páginas que dedica al tema, establece los conceptos y definiciones básicos de la probabilidad, siendo lo más destacable de este texto su anticipación del método de estimación de máxima verosimilitud. En este sentido dice ${ }^{24}$ :

«Si se sabe que en una urna hay cuatro bolas entre blancas y negras, y se han sacado sucesivamente tres bolas blancas y una negra, teniendo cuidado de volver a poner cada vez la bola sacada, podríamos conjeturar que se verificaba alguna de las tres hipótesis siguientes: o que había 3 bolas blancas y 1 negra, o 2 blancas y 2 negras o una blanca y 3 negras.

La última hipótesis es mucho menos probable que las otras dos, porque si la urna contuviese sólo una bola blanca, seria necesario que esta bola hubiese salido tres veces de seguida; y se concibe con facilidad que habría menos dificultad si hubiese dos bolas blancas, y aun menos si hubiese tres.»

\section{LOS INTENTOS DE MODERNIZACIONN}

Los primeros clamores para que se tomaran en cuenta estos nuevos estudios y la aparición de manuales específicos sobre el Cálculo de Probabilidades fueron los pasos necesarios por los que transcurrieron las siguientes décadas, donde sólo unos pocos ilustres profesores abogaban por la puesta al día de nuestra ciencia.

En 1855, Antonio Aguilar y Vela (1821-1882), catedrático de matemáticas y astronomia y secretario perpetuo de la Real Academia de Ciencias Exactas,

\footnotetext{
22 Vallejo, J. M. (1827): Compendio de Matemáticas puras y mistas, t. II, 2." ed., Madrid, Imprenta Garcia.

23 Arnauld, A.; Nicole, P. (1987): La Lógica o el arte de pensar, Madrid, Alfaguara.

24 Vallejo, J. M.: Compendio de Matemáticas, p. 432.
} 
de su grado de doctor en la Facultad de Filosofia (sección de ciencias físicomatemáticas) de la Universidad Central 25 . Dicho discurso se titulaba De la importancia del estudio del Cálculo de Probabilidades, donde, a propósito de la reforma de las enseñanzas universitarias que se avecinaba, reclamaba mayor dedicación a esta materia 26 : «Muchas son las ventajas», decía entonces, «que reportaría al país de la agregación de esta ciencia a alguna de las asignaturas de la sección de ciencias físico-matemáticas, y ya que de algunos años a esta parte se da tan justa importancia al estudio de las últimas, hágase lo mismo con sus principales aplicaciones, y entonces podrá mejor comprenderse la utilidad que en sí encierra el estudio de las ciencias exactas.» No tuvo mucho éxito don Antonio Aguilar, ya que la «Ley Moyano» de 1857, donde se crean las Facultades de Ciencias Exactas, Físicas y Naturales, no contempló ninguna materia estadística en sus planes de estudio ${ }^{27}$.

Por fin se publica en el año 1867 un manual especifícamente dedicado al álgebra probabilistica: Es el Elementos del Cálculo de las Probabilidades del profesor Agustín Martinez Alcibar ${ }^{28}$, donde se queja de «la grande importancia que en España se da a la literatura, comparada con las ciencias exactas y de experimento». Muestra un excelente conocimiento de los trabajos sobre probabilidad y estadística de, prácticamente, todos los autores europeos y, después de exponer los conceptos fundamentales de la probabilidad, se dedica al análisis de un número suficientemente representativo de juegos de azar, estudiando con detenimiento el juego del Monte y el del Bacará. Comenta la aplicación de la Ley de los grandes números e introduce el concepto de esperanza.

El profesor de matemáticas y periodista Felipe Picatoste y Rodríguez (1834-1892) escribió manuales de las más variadas disciplinas. En 1882 edita su Vocabulario matemático-etimológico ${ }^{29}$, donde explica las voces de Cálculo de Probabilidades y probabilidad, confundiendo esta última con la noción actual de ventaja u odd, al establecer que «la probabilidad es el cociente del número de causas favorables por el de las contrarias» ${ }^{30}$. Esta obra tiene un «Breve índice de matemáticos célebres y de sus obras más notables», donde relaciona a

25 Aguilar y Vela, A. (1855): De la importancia del estudio del cálculo de probabilidades, Madrid, Imprenta de Ancos.

26 Aguilar y Vela, A.: De la Importancia, p. 14. Nacional.

28 Martinez Alcibar, A. (1867): Elementos del cálculo de las probabilidades, Madrid, Imprenta J. Fernảndez y compañia.

29 Picatoste y Rodriguez, F. (1862): Vocabulario matemático-etimológico, Madrid, Imprenta

D. E. Aguado.

${ }^{30}$ Picatoste y Rodríguez, F.: Vocabulario matemático-etimológico, p. 95. 
los Bernoulli, a Caramuel, a Condorcet, Fermat, Lagrange, Laplace, De Moivre y a Thomas Simpson con sus respectivas aportaciones en el campo de la probabilidad.

Antes de acabar el siglo XIX, en 1879, el entonces comandante de Artillería Diego Ollero Carmona (1839-1907) publica su Tratado de Cálculo de Probabilidades $^{31}$, que constituye el primer manual moderno en castellano sobre probabilidades donde se hace uso del cálculo diferencial. Este manual se desarrolla en cinco capítulos. En el primero aparece "casi un formulario de las matemáticas necesarias para entender su Tratado" ${ }^{32}$, en el segundo se establecen los principios fundamentales de la probabilidad. El tercero se dedica al teorema de Bernoulli. En el cuarto se estudia la teoria estadística del análisis de errores y en el quinto, y último, se expone el método de los mínimos cuadrados.

\section{LA REAL ACADEMIA DE CIENCIAS COMO VEHICULO DE DIFUSIÓN}

La convicción de que los científicos se deberían reunir para comunicarse los descubrimientos que bien en el pais o bien en el extranjero se fueran realizando, para discutirlos y esclarecer las dudas que se manifestaran entre la colectividad científica, fue el motivo que impulsó la creación de la Real Academia de Ciencias Exactas, Físicas y Naturales en 1847. A lo largo de la existencia de esta Academia, como es lógico, algunos académicos centraron la atención de la asamblea en temas relacionados con la teoría de la probabilidad y sus aplicaciones. Relacionamos, a continuación, todas las intervenciones que se produjeron al respecto.

En 1868, Miguel Merino y Melchor (1831-1905), astrónomo, que dos años antes habia publicado un trabajo titulado Reflexiones y conjeturas sobre la ley de mortalidad en España, en el Discurso de su recepción pública como miembro de la Real Academia de Ciencias Exactas, Físicas y Naturales ${ }^{33}$ analiza con detalle la evolución histórica del Cálculo de Probabilidades, estudiando las principales aportaciones de los cientificos europeos con dos siglos de perspectiva, Arias.

31 Ollero, D. (1913): Tratado de Cálculo de Probabilidad, 4. ed., Madrid, Imprenta Eduardo

32 Sánchez-Lafuente, J. (1975): Historia de la Estadística como Ciencia en España (1500-1900), Madrid, Instituto Nacional de Estadística, p. 268.

${ }_{33}$ Merino, M., y Aguilar, A. (1868): Discursos leidos ante la Real Academia de Ciencias Exactas, Fisicas y Naturales en la recepción pública del Señor Don Miguel Merino, Madrid, Imprenta Aguado. 
y recalca la utilidad de esta ciencia para la mejor conducción de las compañías de seguros.

También empieza a contemplar los primeros conatos de fusión de lo que al principio habíamos denominado ciencia para la elaboración de las estadísticas con el Cálculo de Probabilidades, basándose en los trabajos de Adolphe Quetelet, y recoge la utilidad del método de los minimos cuadrados de Gauss para el análisis de los errores de medición que se detectaban en los trabajos de los astrónomos y geodésicos. Le contestó el académico numerario Antonio Aguilar y Vela, en cuyo discurso recuerda la expresión de que el Cálculo de Probabilidades es «un buen hijo de malos padres», en que se hace referencia a su origen lúdico ${ }^{34}$.

Unos años más tarde, en 1890, en ocasión similar a la anterior, es decir, en el acto de su recepción como académico, Alberto Bosch Fustegueras (1848-1900) disertó sobre las Aplicaciones de las matemáticas a las ciencias morales y políticas ${ }^{35}$, donde, sin excesivo entusiasmo, se refiere al uso de la teoría de la probabilidad en sus aplicaciones al campo actuarial y financiero. Le contestó, con la misma o menor convicción en la falta de solidez de estas teorías, José Echegaray y Eizaguirre (1833-1916), que no tuvo reparó en decir: «y no logran fascinarme ni las cándidas paradojas del caballero de Méré, ni la paradoja llamada de San Petersburgo, que por ser de tierra del Nihilismo y del Pesimismo, debe ser espejo de paradojas, siquiera sea espejo ahumado, ni los errores de Condorcet, ni los hermosos teoremas de Bernoulli, ni los sublimes trabajos de Laplace, ni los de Gauss, ni todas las lucubraciones posteriores, que son todas ellas como esfinges matemáticas, que defienden el templo nebuloso de la diosa Casualidad, la de más veleidades y más coqueterías entre todas las diosas» ${ }^{36}$. El determinismo no dejaba ver a tan ilustre académico la emergencia de una nueva ciencia que se convertiría en un firme apoyo de todas las demás.

En el discurso de recepción de Diego Ollero como académico en 1898 sólo se puede encontrar una alusión puntual a la contribución del «Cálculo de Probabilidades para aquilatar el valor relativo de las armas en lo que toca a su precisión» ${ }^{37}$. Aunque Diego Ollero fue un precursor, como antes quedó establecido, no creyó oportuno aprovechar esta ocasión para difundir sus conoci-

34 Merino, M., y Aguilar, A.: Discursos, p. 94.

35 Bosch, A. (1890): Aplicaciones de las matématicas a las ciencias morales y políticas, Madrid, Real Academia de Ciencias Exactas, Físicas y Naturales.

36 Bosch, A.: Aplicaciones, p. 77.

37 Ollero, D. (1898): Los progresos de las armas de fuego en su relación con las ciencias matemáticas Madrid, Imprenta de L. Aguado. 
mientos sobre la probabilidad y sus aplicaciones, y centró su disertación en la utilización de las matemáticas en el arte de la Artillería.

El ingeniero Pedro M. González Quijano (1870-1958), también en su discurso de ingreso en esta Academia en 1925, disertó sobre Azar y Determinismo ${ }^{38}$ recogiendo las tribulaciones de Laplace y Boole, en línea con las deliberaciones de Bertrand y Poincaré, a quienes no cita. Su definición de probabilidad incorpora la teoria frecuencista concibiéndola, pues, como un re sultado experimental. Le contestó su discurso de ingreso Leonardo de Torres Quevedo (1852-1939), que, al igual que en el caso anterior, hizo una defensa ardiente del determinismo calificando a las verdades experimentales de «contingencia teórica» aunque necesarias en la práctica.

Comprobamos, por tanto, que los aires de la Academia de Ciencias no estaban muy proclives a una actuación amplificadora de estas nuevas ideas.

\section{LA PROBABILIDAD EN LOS TEXTOS DE ESTADÍSTICA}

Por otra parte, en la segunda mitad del siglo XIX la Estadística se había abierto camino en el mundo de la Universidad y sus enseñanzas se simultaneaban con la Economía Política y/o con la Geografia. Comienzan a aparecer cátedras de Economía Política y Estadística en las Facultades de Derecho y de Geografía y Estadística en las Escuelas de Comercio. Fruto de la institucionalización de estas enseñanzas es la publicación de textos sobre Estadística, que de manera concisa citaremos, dado que, en lo referente a la probabilidad, sólo incorporan muy vagos y simples detalles.

En efecto, en 1873, Mariano Carreras y González (1827-1885) y José Manuel Piernas Hurtado (1843-1911) editan su Tratado elemental de Estadística ${ }^{39}$, donde, sin tener muy claro aún qué nexo era el que ligaba la probabilidad con la Estadística, dedican unas páginas a reseñar ideas sobre «probabilidad matemática» y su diferencia con la «probabilidad filosófica» 40 fijando reglas sencillas de la determinación de la primera en algunos ejemplos elementales.

En 1881, Melchor Salvá Hormaechea (1838-1918), en su Tratado elemen-

38 González Quijano, P. M. (1925): Azar y determinismo, Madrid, Talleres Voluntad.

39 Carreras y González, M., y Piernas Hurtado, J. M. (1873): Tratado Elemental de Estadística, Madrid, Imprenta Miguel Guijarro.

40 Carreras y González, M., y Piernas Hurtado, J. M: Tratado, pp. 61.77. 
tal ${ }^{41}$, también intuye la importancia del Cálculo de Probabilidades, citando, incluso, algún pasaje del Ensayo filosófico sobre las probabilidades de Laplace ${ }^{42}$.

Antonio José Pou y Ordinas (1834-1900) publica, en 1889, un Curso de Estadística ${ }^{43}$ donde ya se ofrece una visión más avanzada de la utilidad del Cálculo de Probabilidades en el análisis de datos estadísticos, lo que se nota en bastantes lugares de este manual.

Por fin, y dentro de esta relación de textos básicamente de Estadística, encontramos el que editara en 1897 el citado Piernas Hurtado bajo el titulo de Tratado Elemental de Estadística ${ }^{44}$, donde, en las páginas 93-95, reproduce literalmente la breve referencia que se hacia de la probabilidad en el manual que publicó conjuntamente con el profesor Carreras y González.

\section{LA PRIMERA MITAD DEL SIGLO XX}

Pocos avances notables se producen en nuestro país en el primer tercio del siglo xx en cuanto al progreso del Cálculo de Probabilidades se refiere. A pesar de que la difusión de los textos de Laplace, Lacroix, Liagre, Bertrand, Poincaré y Bachelier es incontestable, no logra efectuarse todavia la revolución probabilística en España. No obstante, reseñaremos algunos autores, pero, fundamentalmente, para mostrar que dicha revolución científica permaneció latente en ese periodo: el texto de Sanpere y Carrera ${ }^{45}$ y el de Nicomedes Alcayde y Carvajal (1871-1930) ${ }^{46}$, émulo del de don Diego Ollero, son una buena prueba de ello.

En 1909 apareció La Gramática de la Ciencia ${ }^{47}$ de Karl Pearson (1857-1936), traducida por Julián Besteiro, donde se analizan los fundamentos metodológicos de la probabilidad, entre otras cuestiones. También en 1909 la Real Academia de Ciencias Exactas, Físicas y Naturales de Madrid convocó un concurso público dedicado a la Exposición clara y sencilla del Cálculo de las Probabilidades.

4t Salvā, M. (1881): Tratado Elemental de Estadística, Madrid, Agustín Jubera.

42 Salvá, M.: Tratado, p. 139.

43 Pou y Ordinas, A. J. (1889): Curso de Estadística, Barcelona, Imprenta Viuda e H. de J. Subirana.

44 Piernas Hurtado, J. M. (1897):Tratado Elemental de Estadística, Madrid. V. Suárez.

45 Sampere y Carrera, J. (1903): Elementos de Algebra, conteniendo el Cálculo de Probabilidades y tablas logarítmicas, Valladolid, Establecimiento tipográfico de F. Santarén Madrazo.

46 Alcayde y Carvajal, N. (1908): Cálculo de las Probabilidades, Guadalajara, Imprenta del Colegio de Huérfanos de la Guerra.

47 Pearson, K. (1909): La Gramática de la Ciencia, Madrid, Daniel Jorro. 
De las Memorias presentadas resultó premiada la de Gabriel Galán Ruiz (1869-?) ${ }^{48}$ y obtuvo accésit la de Manuel Velasco de Pando (18881958) ${ }^{49}$.

En la de Gabriel Galán se expone un resumen bastante completo del estado de la cuestión en ese momento, haciendo especial hincapié en las aplicaciones de la teoría de la probabilidad al cálculo actuarial. En la memoria de Manuel Velasco de Pando también se contemplan los conceptos fundamentales de la teoría de la probabilidad, pero entre las aplicaciones que ofrece, además de dedicar un capítulo íntegro a los seguros, expone la idoneidad del Cálculo de Probabilidades para el análisis de las «jugadas en Bolsa» en un largo epigrafe que subtitula como «aplicaciones a la especulación». Estas Memorias se publicaron al comienzo de los años veinte.

En 1927 se traduce un pequeño manual sobre el Cálculo de Probabilidades, el de Otto Knopf, que tuvo tanto éxito que fue reeditándose sucesivamente durante más de veinte años, y en 1933 aparece el Tratado Elemental de Estadística de Fuentes Martínez, en el que se incorporan algunos capítulos dedicados a esta materia, aunque con una visión más propia de los manuales europeos del siglo anterior ${ }^{50}$.

A lo largo de los años treinta y cuarenta de este siglo se instauraron cátedras de Estadística Matemática en las Facultades de Ciencias Exactas de nuestro pais. La primera, la de Madrid, la obtuvo por oposición Olegario Fernández Baños (1887-1946) en 1933, y empiezan a mostrarse científicos preocupados por la investigación y el desarrollo de la teoría de la probabilidad, consecuencia, en mi opinión, del espíritu regeneracionista de la matemática que impulsó, principalmente, el profesor Julio Rey Pastor (1888-1962); el cual, por otra parte, salvo en algún discurso divulgativo, no se dedicó seriamente al estudio de la Estadística Matemática según se deduce de su numerosísimo catálogo de publicaciones. Algunas aportaciones de Esteban Terradas e Illa (1888-1950) ${ }^{51}$, Sixto Cámara Tecedor (1872-1952) 52, J. María Orts Aracil

48 Galán, G. (1923): Cálculo de las Probabilidades, Madrid, Real Academia de Ciencias Exactas, Físicas y Naturales de Madrid, Gráficas Reunidas, S. A.

t9 Velasco de Pando, M. (1920): Cálculo de las Probabilidades, Madrid, Real Academia de Ciencias Exactas, Fisicas y Naturales de Madrid, Establecimiento tipogräfico de Fortanet.

so Knopf, O. (1927): Cálculo de Probabilidades, Barcelona, Labor. Fuentes Martínez, M. (1933). Tratado Elemental de Estadistica. Madrid, Imp. Juan Pueyo.

"Terradas e Illa, E.: Aunque el profesor Terradas impartió cursos de Estadisca Matemática y de Muestreo en los inicios de la década de los treinta, entre sus numerosas publicaciones no he encontrado ninguna referente a las materias que aqui interesan.

52 Cámara Tecedor, S. (1937): Sobre algunas propiedades elementales de los limites estocásticos, Madrid, Nuevas Gráficas. 
(1891-1968) ${ }^{53}$ y Enrique Cansado Maceda ${ }^{54}$ empiezan a dejarse notar en los círculos universitarios y a aparecer en las revistas científicas españolas.

Pero es a partir de 1945 cuando se dispone de un manual completo de Estadística Matemática: el Tratado de Estadística 55 de Olegario Fernandez Baños; en este manual se contemplan los tópicos clásicos de la estadística descriptiva, de la teoría de la probabilidad y se expone una introducción a la inferencia estadística, rama de la Estadística Matemática que en esas fechas seguía configurándose en el mundo.

El texto incluye una amplísima bibliografía que demuestra que el autor era conocedor del estado de la cuestión en esos momentos, así como de sus antecedentes más notables. Recordemos algunos de los autores que se encuentran en dicha reseña bibliográfica para confirmar esta afirmación. Por orden alfabético: Bachelier, Bayes, Bertrand, Borel, Bortkiewicz, Bowley, Crámer, Czuber, Darmois, Edgeworth, De Finetti, R. A. Fisher, Fréchet, Jeffreys, Kendall, Keynes, Khintchine, Lexis, Liapunoff, Markoff, R. von Misses, Neyman, Pearson (padre e hijo), Poincaré, Student, Tchebycheff, Tschuprow (hijo), Venn, Wald, Wilks, Yates y Yule.

Prologado por el profesor Orts se editó en 1946 otro texto de similares características que el que acabamos de citar: es la Estadística de M. García Álvarez y de J. Gómez Ayuso ${ }^{56}$. Después, además de la edición de la versión española de Enrique Cansado de los Métodos matemáticos de estadística de $\mathrm{H}$. Cramér en $1953{ }^{57}$, vendrian, entre otros, los manuales de los profesores Sixto Ríos, Ubaldo Nieto de Alba y Gonzalo Arnaiz (1916-1990), pero eso ya es el comienzo de la historia moderna de la Estadística Matemática en nuestro país.

\section{EPÍLOGO}

Como conclusión, podemos decir que, si bien no existió en España una verdadera «revolución probabilística», término acuñado por Kruger y otros ${ }^{58}$,

${ }^{3}$ Orts Aracil, J. M. (1941): Iteración de variables aleatorias, Anales de la Asociación española para el progreso de las Ciencias, Madrid.

54 Cansado Maceda, E. (1946): Integral de Stieltjes-Lebesgue y sus aplicaciones a la Estadística, Madrid, Consejo Superior de Investigaciones Cientificas.

35 Fernandez Baños, O. (1945): Tratado de Estadística, Madrid, Consejo Superior de Investigaciones Cientificas.

56 Garcia Álvarez, M., y Ayuso Orejana, J. (1946): Estadística, Madrid, Saeta.

57 Crámer, H. (1953): Métodos matemáticos de estadística, Madrid, Aguilar.

58 Kruger, L., y otros (1987): The probabil the sciences, Cambridge, Mass., MIT Press. 
hasta la segunda mitad de nuestro siglo xx (con casi un siglo de retraso), no podemos olvidar los esfuerzos, aunque esterilizados por la soledad y la incomprensión de los momentos históricos en que les tocó vivir, de todos estos científicos españoles que se anticiparon a la necesidad de fomentar el estudio y la investigación en el campo de la Estadistica Matemática y que en este trabajo, creo que por primera vez, hemos recordado de manera colectiva.

Probablemente este atraso se debió, como señala el profesor Sixto Ríos 59 , a que «el desarrollo de la Ciencia Estadística en España a partir de los comienzos del siglo xx... viene negativamente influenciada por encontrarse su enseñanza adscrita a la Facultad de Derecho hasta 1933, en que se crea la primera Cátedra de Estadística Matemática en la Universidad de Madrid» pero, quizá, la razón última radique en el mismo retraso secular de la Matemática en nuestro pais que tan bien describe José Antonio Artigas (1887-1977) ${ }^{60}$ y analizan Francisco Vera ${ }^{61}$, Juan Vernet ${ }^{62}$ y José María López Piñero ${ }^{63}$. Las causas de este retraso las condensaba el profesor Artigas en los cuatro puntos siguientes:

«1. Que los Matemáticos españoles del siglo XVI en España no habían pasado de la formación de los del siglo xIv.

2. $\quad$ Que desconocían el tesoro algebraico, que otros paises, especialmente Italia, habían recibido ya de la propia España (árabe).

3. Que nuestros historiadores se extraviaron al exaltar a aquellos matemáticos como avanzados e innovadores, cuando sólo eran expositores rezagados.

4. ${ }^{\circ}$ Que la persistencia de esta inferioridad desde mediados del siglo xviII, nos ha hecho entrar en el siglo xx sin haber llegado a tener una cultura matemática moderna» ${ }^{64}$.

59 Ríos, S. (1991): «El progreso de la Ciencia Estadística española en el siglo XX», Estadística Española, núm. 128, Instituto Nacional de Estadistica, Madrid, p. 371.

so Artigas y Sanz, J. A. (1949): Nuestra cultura en la Ciencia (Ciencia Estadística y Genio Hispánico), Real Academia de Ciencias Exactas, Físicas y Naturales, Madrid, Nuevas Gráficas, S. A.

61 Vera, F. (1937): Historia de la Ciencia, Barcelona, Iberia Joaquin Gil, Editor.

62 Vernet Ginés, J. (1975): Historia de la Ciencia Española, Madrid, Instituto de España.

63 López Piñero, J. M. (1979): Ciencia y técnica en la sociedad española de los siglos XVI y XVII, Bar celona, Labor.

ot Artigas y Sanz, J. A: Nuestra cultura en la Ciencia, p. 42. 